\title{
BAIRE SPACES AND GRAPH THEOREMS
}

\author{
DOMINIK NOLL
}

\begin{abstract}
We prove graph theorems and a variant of the Banach-Steinhaus theorem in a purely topological context. We obtain characterizations of Baire spaces in the class of metrizable spaces by means of graph theorems.
\end{abstract}

Introduction. The following abstract form of a graph theorem covers the preponderant part of work in this field:

Let $f$ be a nearly continuous mapping from a space $E$ to a space $F$ which satisfies a certain condition (frequently expressed in terms of the graph of $f$ ). Then, under appropriate conditions on $E$ and $F$, the mapping $f$ is continuous.

The most convenient applications of this scheme deal with the case where the graph of the mapping $f$ is closed. The reader might consult [Kö, p. 33ff], where the classical theory is treated, [Hu] for the situation in topological groups, [Ke], [LR] for the case of uniform spaces, [Wi], [BP], [We] for the general topological case. In this paper, the main interest is directed toward the case where the graph $G(f)$ of $f$ is a $G_{\delta}$-set (more generally a set of interior condensation). In $\$ 1$ we prove that if $E$ is a Baire space and if $F$ is complete (in a certain sense) then the graph theorem above holds for mappings $f$ whose graph is $G_{\delta}$. In $\$ 2$ we prove graph theorems for mappings with measurability conditions. In this case the completeness property for $F$ is no longer needed. As a consequence, in $\$ 2$ we obtain a purely topological variant of the Banach-Steinhaus theorem.

$\$ 3$ is devoted to the study of a converse problem arising from our graph theorems in $\$ \S 1$ and 2. In view of the fact that these theorems hold for source spaces $E$ which are Baire spaces it is natural to pose the following question:

Let $E$ be a space such that a certain graph theorem holds for $E$ and all suitable $f$ and $F$. Must $E$ then be a Baire space?

We prove that the answer is in the positive if $E$ is assumed to be metrizable, thus obtaining three characterizations of Baire spaces in the framework of metrizable topological spaces. Finally we prove that in the absence of metrizability the Baire condition is no longer necessary.

In the final $\$ 4$ we give a brief examination of a graph theorem of Wilhelm's [Wi], in which almost continuous mappings are used instead of the nearly continuous ones used here.

Received by the editors November 7, 1983 and, in revised form, February 4, 1985

1980 Mathematics Subject Classification. Primary 54E52; Secondary 54C10.

Key words and phrases. Complete spaces, Baire spaces, nearly continuous mapping, set of interior condensation, Banach-Steinhaus theorem, graph theorems. 
0. Preliminaries. In this section we give a brief survey of the technical notions used in this paper.

0.1 . Nearly continuous mappings. Let $E, F$ be topological spaces. A mapping $f$ from $E$ to $F$ is called nearly continuous if for every $x \in E$ and every neighborhood $U$ of $f(x)$ the set $\overline{f^{-1}(U)}$ is a neighborhood of $x$. (Compare [Kö, p. 36], [We, Wi, BP].)

0.2. Complete spaces. A topological space $E$ is called complete if there exists a pair $(\phi, T)$ consisting of a tree $T=\left(T, \leqslant_{T}\right)$ (cf. [J, p. 91]) of height $\omega$ and a mapping $\phi$ from $T$ to the topology of $E$ such that the following conditions are satisfied:

(i) the set $\{\phi(t): t \in T\}$ is a base for $E$;

(ii) whenever $t \in T$ then $\left\{\phi(s): t<_{T} s \in T\right\}$ is a base for $\phi(t)$;

(iii) if $b \subset T$ is a cofinal branch [J, p. 91] such that $\phi(t) \neq \varnothing$ for all $t \in b$ then $\bigcap\{\phi(t): t \in b\} \neq \varnothing$, too.

$E$ is called strongly complete if the following condition (iv) holds instead of (iii):

(iv) If $b \subset T$ is a cofinal branch and if $\mathfrak{F}$ is a filter on $E$ with $\phi(t) \in \mathfrak{F}$ for all $t \in b$, then $\mathfrak{F}$ has a cluster point in $\bigcap\{\phi(t): t \in b\}$.

Since (iv) implies (iii), every strongly complete space is complete. In the frame of regular spaces the strongly complete spaces are known under the name 'monotonically Čech complete spaces', used in [C $\check{C} \mathbf{N}]$, and as 'spaces with condition $\mathscr{K}$ ', used in [WW]. The class of complete spaces is closed under arbitrary products and is $G_{\delta}$-hereditary. Every complete space is Baire.

0.3. Sets of interior condensation. A subset $P$ of a topological space $E$ is called a set of interior condensation in $E$ if there exists a pair $(\phi, T)$ consisting of a tree $T$ of height $\omega$ and a mapping $\phi$ from $T$ to the topology of $E$ such that the following conditions are satisfied:

(i) $\{\phi(t): t \in T\}$ is a cover of $P$;

(ii) whenever $t \in T$ then $\left\{\phi(s): t<_{T} s \in T\right\}$ covers $P \cap \phi(t)$;

(iii) if $b \subset T$ is a cofinal branch, then $\bigcap\{\phi(t): t \in b\} \subset P$.

Every $G_{\delta}$-set is a set of interior condensation. The concept of a set of interior condensation has been introduced by Wicke and Worrell in [WW]. We have reproduced their definition here in a slightly modified form.

0.4. The Banach-Mazur game. We will use here the classical Banach-Mazur game between players A and B both playing with perfect information (see [Ox], [Wh]). A strategy for player $\mathrm{A}$ is a mapping $\alpha$ whose domain is the set of all decreasing sequences $\left(G_{1}, \ldots, G_{2 n-1}\right), n \geqslant 1$, of nonempty open sets such that $\alpha\left(G_{1}, \ldots, G_{2 n-1}\right)$ is a nonempty open set contained in $G_{2 n-1}$. Dually, a strategy for player B is a mapping $\beta$ whose domain is the set of all decreasing sequences $\left(U_{1}, \ldots, U_{2 n}\right), n \geqslant 0$, of nonempty open sets such that $\beta\left(U_{1}, \ldots, U_{2 n}\right)$ is nonempty, open and contained in $U_{2 n}$. Here $n=0$ stands for the empty sequence, for which $\beta(\varnothing)$ is nonempty and open, too. If $\alpha, \beta$ are strategies for A, B respectively, then the unique sequence $G_{1}$, $G_{2}, G_{3}, \ldots$ defined by $\beta(\varnothing)=G_{1}, \alpha\left(G_{1}\right)=G_{2}, \beta\left(G_{1}, G_{2}\right)=G_{3}, \alpha\left(G_{1}, G_{2}, G_{3}\right)=$ $G_{4}, \ldots$ is called the game of A with $\alpha$ against B with $\beta$. We will say that A with $\alpha$ wins against B with $\beta$ if $\cap\left\{G_{n}: n \in \mathbf{N}\right\} \neq \varnothing$ holds for the game $G_{1}, G_{2}, \ldots$ of A with $\alpha$ against B with $\beta$. Conversely, we will say that B with $\beta$ wins against $\mathrm{A}$ with $\alpha$ if $\mathrm{A}$ with $\alpha$ does not win against B with $\beta$. 
We will make use of the following theorem, essentially proved by Banach and Mazur (cf. [Ox]):

Let $E$ be a topological space. The following are equivalent:

(1) $E$ is a Baire space;

(2) for every strategy $\beta$ of B there exists a strategy $\alpha$ of A such that A with $\alpha$ wins against $\mathrm{B}$ with $\beta$.

1. The main theorems. In this section we prove our fundamental results.

THEOREM 1. Let E be a Baire space and let $F$ be a regular complete space. Suppose that $f$ is a nearly continuous mapping from $E$ to $F$ whose graph $G(f)$ is a set of interior condensation in $E \times F$. Then $f$ is continuous.

Proof. Let $(\phi, T)$ be given on $F$ as in 0.2 and let $(\psi, C)$ be given for the graph $G(f)$ of $f$ as in 0.3. Fix $x \in E$ and a neighborhood $U$ of $f(x)$. Choose, by the regularity of $F$, an open neighborhood $V$ of $f(x)$ with $\bar{V} \subseteq U$. We wish to prove int $\overline{f^{-1}(V)} \subseteq f^{-1}(U)$. Take $y \in \operatorname{int} \overline{f^{-1}(V)}$. It is sufficient to prove $f(y) \in \bar{V}$. To this end, we take an open neighborhood $W$ of $f(y)$. If we can establish $V \cap W \neq \varnothing$, the proof will be finished.

We will now define a strategy $\beta$ for player $B$ in the game described in 0.4 . For this purpose it will be helpful to have well-orders on the sets $T, C, E$ and $\mathfrak{U}$, where $\mathfrak{U}$ is the set of all open boxes $U \times V$ in $E \times F$. Now we can start.

(1) $\beta(\varnothing)$ has to be defined. Since int $\overline{f^{-1}(W)}$ is a neighborhood of $y \in \overline{f^{-1}(V)}$, there exists a first (for the well-order of $E$ ) element $z_{1} \in \operatorname{int} \overline{f^{-1}(W)} \cap f^{-1}(V)$. By property (i) in 0.2 there exists a first (for the well-order of $T$ ) element $t_{1} \in T$ with $f\left(z_{1}\right) \in \phi\left(t_{1}\right) \subseteq V$. Now $\left(z_{1}, f\left(z_{1}\right)\right) \in G(f)$ and, consequently, by (i) in 0.3 there exists a first (for the well-order of $C$ ) $b_{1} \in C$ with $\left(z_{1}, f\left(z_{1}\right)\right) \in \psi\left(b_{1}\right)$. Finally, there exists a first (for the well-order of $\mathfrak{u}$ ) $U_{1} \times V_{1}$ such that $\left(z_{1}, f\left(z_{1}\right)\right) \in U_{1} \times V_{1} \subseteq$ $\psi\left(b_{1}\right), \quad U_{1} \subseteq \operatorname{int} \overline{f^{-1}(W)}, \quad V_{1} \subseteq \phi\left(t_{1}\right)$. Observe now that $U_{1} \cap \operatorname{int} \overline{f^{-1}\left(V_{1}\right)}$ is a neighborhood of $z_{1} \in \overline{f^{-1}(W)}$ and therefore we find a first $y_{1} \in U_{1} \cap$ int $\overline{f^{-1}\left(V_{1}\right)}$ $\cap f^{-1}(W)$. Choose the first $s_{1} \in T$ with $f\left(y_{1}\right) \in \phi\left(s_{1}\right) \subseteq W$ and the first $c_{1} \in C$ with $\left(y_{1}, f\left(y_{1}\right)\right) \in \psi\left(c_{1}\right)$. Then take the first $O_{1} \times W_{1}$ in $\mathfrak{U}$ with $\left(y_{1}, f\left(y_{1}\right)\right) \in O_{1} \times$ $W_{1} \subseteq \psi\left(c_{1}\right), O_{1} \subseteq U_{1} \cap$ int $\overline{f^{-1}\left(V_{1}\right)}$ and $W_{1} \subseteq \phi\left(s_{1}\right)$. Now we define $\beta(\varnothing):=O_{1} \cap$ int $\overline{f^{-1}\left(W_{1}\right)}$.

(2) $\beta\left(G_{1}, G_{2}\right)$ has to be defined, where $G_{1}, G_{2}$ are nonempty and open with $G_{2} \subseteq G_{1}$. Examine whether $\beta(\varnothing)=G_{1}$. If not, then we may define $\beta\left(G_{1}, G_{2}\right)$ as we like, say $\beta\left(G_{1}, G_{2}\right)=G_{2}$. If $\beta(\varnothing)=G_{1}$ then $G_{1}=O_{1} \cap$ int $\overline{f^{-1}\left(W_{1}\right)}$, i.e. the construction of (1) above is available. Hence $G_{2} \subseteq O_{1} \cap$ int $\overline{f^{-1}\left(W_{1}\right)}$ and, since $O_{1} \subseteq$ int $\overline{f^{-1}\left(V_{1}\right)}$, there exists a first (for the well-order of $\left.E\right) z_{2} \in G_{2} \cap f^{-1}\left(V_{1}\right)$. Now we have $f\left(z_{2}\right) \in V_{1} \subseteq \phi\left(t_{1}\right)$. Hence, by condition (ii) in 0.2 , there exists $t_{2} \in T$ with $t_{1}<{ }_{T} t_{2}$ such that $f\left(z_{2}\right) \in \phi\left(t_{2}\right) \subseteq V_{1}$ and, in addition, $t_{2}$ is the first for the well-order of $T$ with this property. But now we have $\left(z_{2}, f\left(z_{2}\right)\right) \in O_{1} \times V_{1} \subseteq U_{1} \times$ $V_{1} \subseteq \psi\left(b_{1}\right)$. Hence, by condition (ii) in 0.3 there exists a first (for the well-order of $C$ ) element $b_{2} \in C$ with $b_{1}<_{C} b_{2}$ and $\left(z_{2}, f\left(z_{2}\right)\right) \in \psi\left(b_{2}\right)$. Let $U_{2} \times V_{2}$ be the first element of $\mathfrak{U}$ such that $\left(z_{2}, f\left(z_{2}\right)\right) \in U_{2} \times V_{2} \subseteq \psi\left(b_{2}\right), U_{2} \subseteq G_{2}$ and $V_{2} \subseteq \phi\left(t_{2}\right)$. 
Now $U_{2} \cap \operatorname{int} \overline{f^{-1}\left(V_{2}\right)}$ is a neighborhood of $z_{2} \in G_{2} \subseteq \overline{f^{-1}\left(W_{1}\right)}$. Hence there exists a first $y_{2} \in U_{2} \cap \operatorname{int} \overline{f^{-1}\left(V_{2}\right)} \cap f^{-1}\left(W_{1}\right)$. Let $s_{2} \in T$ be the first (for the well-order of $T$ ) such that $s_{1}<_{T} s_{2}$ and $f\left(y_{2}\right) \in \phi\left(s_{2}\right) \subseteq W_{1}$, which exists by (ii) in 0.2 and the fact that $W_{1} \subseteq \phi\left(s_{1}\right)$. Let $c_{2} \in C$ be the first with $c_{1}<_{C} c_{2}$ and $\left(y_{2}, f\left(y_{2}\right)\right) \in \psi\left(c_{2}\right)$, which exists since $\left(y_{2}, f\left(y_{2}\right)\right) \in U_{2} \times W_{1} \subseteq O_{1} \times W_{1} \subseteq \psi\left(c_{1}\right)$. Finally, let $O_{2} \times W_{2}$ be the first element of $\mathfrak{U}$ with $\left(y_{2}, f\left(y_{2}\right)\right) \in O_{2} \times W_{2} \subseteq \psi\left(c_{2}\right)$, $W_{2} \subseteq \phi\left(s_{2}\right), O_{2} \subseteq U_{2} \cap$ int $\overline{f^{-1}\left(V_{2}\right)}$ and then define $\beta\left(G_{1}, G_{2}\right):=O_{2} \cap$ int $\overline{f^{-1}\left(W_{2}\right)}$.

(3) In this way we proceed to define $\beta$ by recursion. At every stage we examine whether the sequence $\left(G_{1}, \ldots, G_{2 n}\right)$ has been defined 'along our route'. If not, then the next move will be of no importance, i.e. we may define $\beta\left(G_{1}, \ldots, G_{2 n}\right)=G_{2 n}$. But if $\beta(\varnothing)=G_{1}$ and $\beta\left(G_{1}, \ldots, G_{2 k}\right)=G_{2 k+1}$ for all $k<n$, then all the former steps are available and we can define $G_{2 n+1}$ in analogy with (2).

Suppose now that $\beta$ has been defined. Since $E$, by assumption, is a Baire space, there exists a strategy $\alpha$ for player A such that A with $\alpha$ wins against B with $\beta$ (see $0.4)$. Let $G_{1}, G_{2}, \ldots$ be the game of $\mathrm{A}$ with $\alpha$ against $\mathrm{B}$ with $\beta$. Then, by the definition of $\beta$, there exist sequences $\left(U_{n}\right),\left(V_{n}\right),\left(O_{n}\right),\left(W_{n}\right),\left(t_{n}\right),\left(s_{n}\right),\left(b_{n}\right),\left(c_{n}\right)$ and $\left(y_{n}\right),\left(z_{n}\right)$ such that the following conditions are satisfied:

(1) $G_{2 n-1}=O_{n} \cap \operatorname{int} \overline{f^{-1}\left(W_{n}\right)}$ and $G_{2 n} \subset G_{2 n-1}$;

(2) $z_{n+1}$ is the first (for the well-order on $E$ ) element of $G_{2 n} \cap f^{-1}\left(V_{n}\right)$;

(3) $t_{n+1}$ is the first (for the well-order on $T$ ) element of $T$ with $t_{n}<_{T} t_{n+1}$ and $f\left(z_{n+1}\right) \in \phi\left(t_{n+1}\right) \subset V_{n}$;

(4) $b_{n+1}$ is the first element of $C$ with $b_{n}<_{C} b_{n+1},\left(z_{n+1}, f\left(z_{n+1}\right)\right) \in \psi\left(b_{n+1}\right)$;

(5) $U_{n+1} \times V_{n+1}$ is the first element of $\mathfrak{u}$ with $z_{n+1} \in U_{n+1} \subset G_{2 n}, f\left(z_{n+1}\right) \in$ $V_{n+1} \subset \phi\left(t_{n+1}\right)$ and $U_{n+1} \times V_{n+1} \subset \psi\left(b_{n+1}\right)$;

(6) $y_{n+1}$ is the first element of $U_{n+1} \cap$ int $\overline{f^{-1}\left(V_{n+1}\right)} \cap f^{-1}\left(W_{n}\right)$;

(7) $s_{n+1} \in T$ is the first with $s_{n}<_{T} s_{n+1}, f\left(y_{n+1}\right) \in \phi\left(s_{n+1}\right) \subset W_{n}$;

(8) $c_{n+1 C}>c_{n}$ is the first with $\left(y_{n+1}, f\left(y_{n+1}\right)\right) \in \psi\left(c_{n+1}\right)$;

(9) $O_{n+1} \times W_{n+1}$ is the first in $\mathfrak{U}$ with $y_{n+1} \in O_{n+1} \subset U_{n+1} \cap \operatorname{int} \overline{f^{-1}\left(V_{n+1}\right)}$, $f\left(y_{n+1}\right) \in W_{n+1} \subset \phi\left(s_{n+1}\right)$ and $O_{n+1} \times W_{n+1} \subset \psi\left(c_{n+1}\right)$;

(10) $G_{2 n+1}=O_{n+1} \cap \operatorname{int} \overline{f^{-1}\left(W_{n+1}\right)}$.

By (1), (5) and (9) we have $\bigcap\left\{G_{n}: n \in \mathbf{N}\right\}=\bigcap\left\{U_{n}: n \in \mathbf{N}\right\}=\bigcap\left\{O_{n}: n \in \mathbf{N}\right\}$. But note that $\alpha$ is winning, hence this intersection is nonempty. Choose $u$ herein. By the definition of completeness (see 0.2) there exist elements $v \in \bigcap\left\{\phi\left(t_{n}\right): n \in \mathbf{N}\right\}$ and $w \in \bigcap\left\{\phi\left(s_{n}\right): n \in \mathbf{N}\right\}$, both by condition (iii) in 0.2. By (5) and (3) we have $(u, v) \in U_{n} \times V_{n} \subset \psi\left(b_{n}\right)$ for all $n$, which yields $(u, v) \in G(f)$ in view of condition (iii) in 0.3. Hence, $v=f(u)$. A similar argument using (9) and (7) yields $(u, w) \in$ $G(f)$, hence $w=f(u)$, hence $v=w$. But note that $v \in \phi\left(t_{1}\right) \subset V$ and $w \in \phi\left(s_{1}\right) \subset$ $W$. Thus $V \cap W \neq \varnothing$ is established and the proof is finished.

The following closed graph theorem essentially uses the same method of proof.

THEOREM 2. Let $E$ be a Baire space and let $F$ be a regular strongly complete space (i.e. a monotonically Čech complete space). Suppose that $f$ is a nearly continuous mapping from $E$ to $F$ whose graph is closed. Then $f$ is continuous. 
Proof. Let $(\phi, T)$ be given for $F$ as in 0.2 with condition (iv) instead of (iii). Now let $U, V, W$ and $x, y$ have the same meaning as in the proof of Theorem 1. Again we have to prove $V \cap W \neq \varnothing$. To this end we define a strategy $\beta$ for player B in the Banach-Mazur game.

(1) $\beta(\varnothing)$ has to be defined. We have int $\overline{f^{-1}(V)} \cap$ int $\overline{f^{-1}(W)} \neq \varnothing$. Hence we

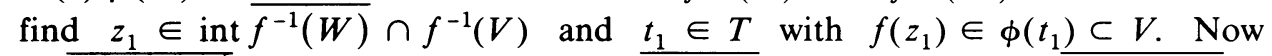
int $\overline{f^{-1}\left(\phi\left(t_{1}\right)\right)}$ is a neighborhood of $z_{1} \in \overline{f^{-1}(W)}$. Hence we have int $\overline{f^{-1}\left(\phi\left(t_{1}\right)\right)} \cap$ $f^{-1}(W) \neq \varnothing$. Choose $y_{1}$ herein. Take $s_{1} \in T$ with $f\left(y_{1}\right) \in \phi\left(s_{1}\right) \subset W$. Define $\beta(\varnothing):=$ int $\overline{f^{-1}\left(\phi\left(t_{1}\right)\right)} \cap$ int $\overline{f^{-1}\left(\phi\left(s_{1}\right)\right)}$.

(2) $\beta\left(G_{1}, G_{2}\right)$ has to be defined. If $G_{1} \neq \beta(\varnothing)$ then define $\beta\left(G_{1}, G_{2}\right):=G_{2}$. Assume $\beta(\varnothing)=G_{1}$. Thus the construction of (1) is available. Choose $z_{2} \in G_{2} \cap$ $f^{-1}\left(\phi\left(t_{1}\right)\right)$. By (ii) in 0.2 choose $t_{2} \in T$ with $t_{1}<_{T} t_{2}$ and $f\left(z_{2}\right) \in \phi\left(t_{2}\right)$. Now int $\overline{f^{-1}\left(\phi\left(t_{2}\right)\right)}$ is a neighborhood of $z_{2} \in G_{2} \subset \overline{f^{-1}\left(\phi\left(s_{1}\right)\right)}$. Hence there exists $y_{2} \in G_{2} \cap$ int $\overline{f^{-1}\left(\phi\left(t_{2}\right)\right)} \cap f^{-1}\left(\phi\left(s_{1}\right)\right)$. Let $\underline{s_{2} T}>s_{1}$ be an element of $T$ with $f\left(y_{2}\right)$ $\in \phi\left(s_{2}\right)$. Now define $\beta\left(G_{1}, G_{2}\right):=G_{2} \cap$ int $\overline{f^{-1}\left(\phi\left(t_{2}\right)\right)} \cap$ int $\overline{f^{-1}\left(\phi\left(s_{2}\right)\right)}$.

This process may be continued in analogy with the proof of Theorem 1 in order to obtain the strategy $\beta$.

Suppose that $\beta$ has been defined. Since $E$ is a Baire space there exists a strategy $\alpha$ for A such that A with $\alpha$ wins against B with $\beta$. Let $G_{1}, G_{2}, \ldots$ be the game of A with $\alpha$ against B with $\beta$. By the construction of $\beta$ there exist sequences $\left(t_{n}\right),\left(s_{n}\right)$ and $\left(y_{n}\right),\left(z_{n}\right)$ such that the following conditions are satisfied:

(1) $z_{n+1} \in G_{2 n} \subset$ int $\overline{f^{-1}\left(\phi\left(t_{n}\right)\right)} \cap$ int $\overline{f^{-1}\left(\phi\left(s_{n}\right)\right)}, f\left(z_{n+1}\right) \in \phi\left(t_{n+1}\right)$ and $t_{n}$ ${ }_{T} t_{n+1}, \phi\left(t_{1}\right) \subset V$ $W$;

(2) $y_{n+1} \in G_{2 n} \cap \operatorname{int} \overline{f^{-1}\left(\phi\left(t_{n+1}\right)\right)}, f\left(y_{n+1}\right) \in \phi\left(s_{n+1}\right)$ and $s_{n}<_{T} s_{n+1}, \phi\left(s_{1}\right) \subset$

(3) $G_{2 n+1}=G_{2 n} \cap \operatorname{int} \overline{f^{-1}\left(\phi\left(t_{n+1}\right)\right)} \cap \operatorname{int} \overline{f^{-1}\left(\phi\left(s_{n+1}\right)\right)}$.

Now we find $u \in \bigcap\left\{G_{n}: n \in \mathbf{N}\right\}$. Let $\mathfrak{M}$ be the set of all pairs $(N, k)$ where $N$ is a neighborhood of $u$ contained in $G_{2 k+1}$. For $(N, n) \in \mathfrak{M}$ we have $N \cap f^{-1}\left(\phi\left(t_{n}\right)\right) \neq$ $\varnothing$ and $N \cap f^{-1}\left(\phi\left(s_{n}\right)\right) \neq \varnothing$ by conditions (1) and $G_{2 n+1} \subset G_{2 n}$. Choose $x(N, n) \in$ $f^{-1}\left(\phi\left(t_{n}\right)\right)$ and $y(N, n) \in f^{-1}\left(\phi\left(s_{n}\right)\right)$ with $x(N, n), y(N, n) \in N$. Suppose that $(N, i) \prec\left(N^{\prime}, i^{\prime}\right)$ holds if and only if $N^{\prime} \subset N$ and $i^{\prime} \geqslant i$. Obviously, the nets $\langle x(N, i)\rangle$ and $\langle y(N, i)\rangle$ both converge to $u$. On the other hand, the net $\langle f(x(N, i))\rangle$ has a cluster point $v \in \bigcap\left\{\phi\left(t_{n}\right): n \in \mathbf{N}\right\} \subset V$ and the net $\langle f(y(N, i))\rangle$ has a cluster point $w \in \bigcap\left\{\phi\left(s_{n}\right): n \in \mathbf{N}\right\} \subset W$. Since the graph $G(f)$ of $f$ is closed we conclude $(u, v) \in G(f)$ and $(u, w) \in G(f)$. Hence $v=w$. This proves $V \cap W \neq \varnothing$.

2. Applications. In this section we prove two consequences of Theorem 1. Theorem 4 below may be regarded as a variant of the Banach-Steinhaus theorem.

The first result is obtained for mappings which are Borel measurable of class one. Recall that $f: E \rightarrow F$ is called Borel measurable of class one if the preimages of open sets are $F_{\sigma}$-sets. We have the following proposition:

Let $f$ be a mapping from a topological space $E$ to a regular $\theta$-refinable ( $c f$. [WW1]) space $F$ whose diagonal $\Delta_{F}$ is a $G_{\delta}$-set in $F \times F$. Suppose that $f$ is Borel measurable of class one. Then f has a $G_{\delta^{-}}$-graph in $E \times F$. 
Proof. Since $F$ is regular and has a $G_{\delta}$-diagonal, there exists a sequence $\left(\mathfrak{U}_{n}\right.$ : $n \in \mathbf{N}$ ) of open covers of $F$ such that $x \in U_{n} \in \mathfrak{U}_{n}$ for $n \in \mathbf{N}$ implies $\cap\left\{\bar{U}_{n}: n \in \mathbf{N}\right\}=\{x\}$ (see [Ce]). By the definition of $\theta$-refinability [WW1] there exist sequences $\left(\mathfrak{U}_{n, m}: m \in \mathbf{N}\right)$ of open covers of $F$ such that $\mathfrak{U}_{n, m}$ refines $\mathfrak{U}_{n}$ and for every $n \in \mathbf{N}$ and every $x \in F$ there exists $m(n, x) \in \mathbf{N}$ such that $x$ is in at most finitely many elements of $\mathfrak{U}_{n, m(n, x)}$. By assumption, the sets $f^{-1}(\bar{U}), U \in \mathfrak{U}_{n, m}$ are $G_{\delta}$. Hence we can find open sets $G(U, 1) \supset G(U, 2) \supset \cdots$ with $\bigcap\{G(U, k): k \in \mathbf{N}\}$ $=f^{-1}(\bar{U})$. Define $V(n, m, k):=\bigcup\left\{G(U, k) \times U: U \in \mathfrak{U}_{n, m}\right\}$. Then we have $\operatorname{graph}(f)=\bigcap\{V(n, m, k): n, m, k \in \mathbf{N}\}$.

REMARK 1. If preimages of open sets are $G_{\delta}$-sets, then the same proof works if we choose open sets $G(U, 1) \supset G(U, 2) \supset \cdots$ with $\bigcap\{G(U, k): k \in \mathbf{N}\}=f^{-1}(U)$. In this case the regularity of $F$ is not needed. A mapping with this property will be called $G_{\delta}$-continuous.

Now we obtain the following consequence of Theorem 1 and the proposition above:

THEOREM 3. Let $E$ be a Baire space and let $F$ be a subspace of the product of $a$ family of regular $\theta$-refinable complete spaces $F_{i}, i \in I$, where each $F_{i}$ has $a G_{\delta}$-diagonal. Then every nearly continuous mapping f from $E$ to $F$ which is either Borel measurable of class one or $G_{\delta}$-continuous is in fact continuous.

Proof. Let $F$ be embedded into the product $\Pi\left\{F_{i}: i \in I\right\}$. If $p_{i}$ denotes the projection onto the $i$ th coordinate space, then we have to prove the continuity of $p_{i} \circ f, i \in I$. Each $p_{i} \circ f$ is nearly continuous and Borel measurable of class one (respectively $G_{\delta}$-continuous). Hence the $p_{i} \circ f$ are continuous by the results above together with Theorem 1.

COROLlARY. Let $E$ be a Baire space and let $F$ be a completely regular space. Then every nearly continuous mapping $f$ from $E$ to $F$ which is either Borel measurable of class one or $G_{\delta}$-continuous, is in fact continuous.

Proof. Every completely regular space is a subspace of a product of lines.

RemarK 2. A mapping $f: E \rightarrow F$ which is the pointwise limit of a sequence $\left(f_{n}\right)$ of continuous mappings is called 'of the Baire class one'. Now if $F$ is completely regular then, for every $g \in C(F, \mathbf{R}), g \circ f$ is again of the first Baire class and, consequently, is Borel measurable of class one. Indeed, if $C \subset \mathbf{R}$ is closed there exist open sets $G_{1}$, $G_{2}, \ldots$ with $\bar{G}_{n+1} \subset G_{n}$ and $C=\bigcap\left\{G_{n}: n \in \mathbf{N}\right\}$. But now we have

$$
f^{-1}\left(g^{-1}(C)\right)=\bigcap\left\{\bigcup\left\{f_{k}^{-1}\left(g^{-1}\left(G_{n}\right)\right): k \geqslant m\right\}: n, m \in \mathbf{N}\right\} .
$$

Using this we could derive another graph theorem for nearly continuous mappings of the first Baire class from Theorem 3. However, it turns out that there is a direct way to obtain an application of Theorem 1 to mappings of the first Baire class not using Theorem 3.

We have the following proposition:

Let $f$ be a mapping from a space $E$ to a regular space $F$ whose diagonal $\Delta_{F}$ is a set of interior condensation in $F \times F$. Suppose that $f$ is the pointwise limit of a sequence $\left(f_{n}\right)$ 
of continuous mappings. Then the graph $G(f)$ of $f$ is a set of interior condensation in $E \times F$.

Proof. Let $(\phi, T)$ be given for the diagonal of $F$ as in 0.3 . Since $F \times F$ is regular we may assume that $t^{\prime}<_{T} t$ implies $\overline{\phi(t)} \subset \phi\left(t^{\prime}\right)$. In fact, this is an argument quite familiar in the base of countable order theory as expounded in [WW] and subsequent papers of the same authors. Now let $B$ be the set of all sequences $\left(\left(n_{1}, t_{1}\right), \ldots,\left(n_{k}, t_{k}\right)\right)$ with $n_{1}<n_{2}<\cdots<n_{k}$ in $\mathbf{N}$ and $t_{1}<_{T} t_{2}<_{T} \cdots<_{T} t_{k}$. Hence $B$ is a tree of height $\omega$ if we assume that $\left(\left(n_{1}, t_{1}\right), \ldots,\left(n_{k}, t_{k}\right)\right) \prec$ $\left(\left(m_{1}, s_{1}\right), \ldots,\left(m_{r}, s_{r}\right)\right)$ holds if and only if $k \leqslant r$ and $\left(n_{i}, t_{i}\right)=\left(m_{i}, s_{i}\right)$ for $i \leqslant k$. Now define $\psi$ by

$$
\begin{aligned}
\psi\left(\left(n_{1}, t_{1}\right), \ldots,\left(n_{k}, t_{k}\right)\right) & \\
& :=\left\{(x, y) \in E \times F:(y, y) \in \phi\left(t_{k}\right),\left(f_{n_{j}}(x), y\right) \in \phi\left(t_{j}\right) \text { for } j \leqslant k\right\} .
\end{aligned}
$$

Obviously, these are open sets. We have to check properties (i)-(iii) in 0.3. We prove (ii) and (iii). Assume $(x, f(x)) \in \psi\left(\left(n_{1}, t_{1}\right), \ldots,\left(n_{k}, t_{k}\right)\right)$. Hence $(f(x), f(x)) \in$ $\phi\left(t_{k}\right)$. By (ii) in 0.3 there exists $t_{k+1}>{ }_{T} t_{k}$ such that $(f(x), f(x)) \in \phi\left(t_{k+1}\right)$. But $\phi\left(t_{k+1}\right)$ is open, and therefore there exists $n_{k+1}>n_{k}$ such that $\left(f_{n_{k+1}}(x), f(x)\right) \in$ $\phi\left(t_{k+1}\right)$, hence $(x, f(x)) \in \psi\left(\left(n_{1}, t_{1}\right), \ldots,\left(n_{k+1}, t_{k+1}\right)\right)$. This proves (ii). To prove (iii) assume that $(x, y) \in \psi\left(\left(n_{1}, t_{1}\right), \ldots,\left(n_{k}, t_{k}\right)\right)$ for $k \in \mathbf{N}$. This yields $\left(f_{n_{j}}(x), y\right)$ $\in \phi\left(t_{j}\right) \subset \overline{\phi\left(t_{j}\right)} \subset \phi\left(t_{k}\right)$ for $j>k$, hence $(f(x), y) \in \phi\left(t_{k}\right)$. This implies $(f(x), y)$ $\in \Delta_{F}$.

In connection with Theorem 1 this yields the announced Banach-Steinhaus type theorem. Note that the class of spaces $F$ for which this theorem holds is again very large. In particular, it contains all completely regular spaces.

THEOREM 4. Let $E$ be a Baire space and let $F$ be a subspace of a product of regular complete spaces each having a diagonal which is a set of interior condensation. Then every nearly continuous mapping $f$ from $E$ to $F$ which is the pointwise limit of a sequence of continuous mappings is in fact continuous.

Proof. The same argument as in the proof of Theorem 3 applies.

REMARK 3. In the classical antecedents of this theorem one can, in addition, give some information on the 'degree of convergence' of the sequence $\left(f_{n}\right)$ under consideration. Namely, one can prove that the convergence is uniform on every compact subset of $E$. The reader will, however, convince himself that this is not possible in the general topological case. Counterexamples can easily be given in $\mathbf{R}$.

3. Converse theorems. In this section we pose the following question. Let $E$ be a topological space for which the statements of Theorem 1, respectively of Theorems 3 or 4 , hold true. Must $E$ then be a Baire space? It turns out that the answer to this question is in the positive if $E$ is assumed to be metrizable. However, if $E$ is only assumed to be Hausdorff, then the answer is in the negative, i.e. there exist first category Hausdorff spaces for which the statements of the graph theorems in $\S \S 1$ and 2 hold true. 
THEOREM 5. Let E be a metrizable topological space. The following statements are equivalent:

(1) $E$ is a Baire space;

(2) every nearly continuous real-valued mapping $f$ on $E$ which is Borel measurable of class one is continuous;

(3) every nearly continuous real-valued mapping $f$ on $E$ whose graph is a $G_{\delta}$-set is continuous;

(4) every nearly continuous real-valued mapping $f$ on $E$ which is the pointwise limit of a sequence of continuous mappings is itself continuous.

Proof. (1) implies (2), (3) and (4) by Theorems 3, 1 and 4, respectively. (3) implies (2) since every $f: E \rightarrow \mathbf{R}$ which is Borel measurable of class one has a $G_{\boldsymbol{\delta}}$-graph. Indeed, define $\varphi: E \times \mathbf{R} \rightarrow \mathbf{R}$ by $\varphi(x, y):=|f(x)-y|$, then $\varphi$ is Borel measurable of class one and therefore $G(f)=\varphi^{-1}(0)$ is a $G_{\delta}$-set. (4) implies (2) by [Ku, Theorem 7 , p. 391] where it is proved that a real-valued mapping $f$ on a metrizable space $E$ which is Borel measurable of class one is in fact the pointwise limit of a sequence of continuous mappings. Proving that (2) implies (1) remains. This will be done in two steps.

(I) First we show that every separable nonempty open subset of $E$ must be of the second category. Assume not. Hence there exists a separable nonempty open subset $U$ of $E$ which is of the first category. Since $E$ is metrizable, there exists a sequence $C_{1}, C_{2}, \ldots$ of nowhere dense closed sets with $C_{1} \subset C_{2} \subset \cdots$ and $\bigcup\left\{C_{n}: n \in \mathbf{N}\right\}=U$. We will construct a sequence $\left(P_{n}: n \in \mathrm{N}\right)$ of mutually disjoint $F_{\sigma}$-sets $P_{n}$ such that $\bigcup\left\{P_{n}: n \in \mathbf{N}\right\}=U$ and $\bar{P}_{n}=\bar{U}$ for all $n \in \mathbf{N}$. Let $\left\{G_{n}: n \in \mathbf{N}\right\}$ be a base for $U$. By induction we define a sequence $n(1), n(2), \ldots$ of natural numbers with $n(i)<$ $n(i+1)$ such that the following relations hold:

$$
\begin{aligned}
& C_{n(1)} \cap G_{1} \neq \varnothing, \\
& \left(C_{n(2)} \backslash C_{n(1)}\right) \cap G_{2} \neq \varnothing, \quad\left(C_{n(3)} \backslash C_{n(2)}\right) \cap G_{2} \neq \varnothing,
\end{aligned}
$$

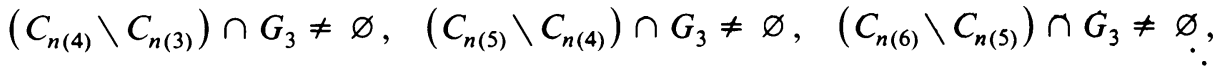

etc.

$$
\begin{aligned}
& P_{1}:=C_{n(1)} \cup\left(C_{n(2)} \backslash C_{n(1)}\right) \cup\left(C_{n(4)} \backslash C_{n(3)}\right) \cup\left(C_{n(7)} \backslash C_{n(6)}\right) \cup \cdots, \\
& P_{2}:=\left(C_{n(3)} \backslash C_{n(2)}\right) \cup\left(C_{n(5)} \backslash C_{n(4)}\right) \cup\left(C_{n(8)} \backslash C_{n(7)}\right) \cup \cdots, \\
& P_{3}:=\left(C_{n(6)} \backslash C_{n(5)}\right) \cup\left(C_{n(9)} \backslash C_{n(8)}\right) \cup\left(C_{n(13)} \backslash C_{n(12)}\right) \cup \cdots,
\end{aligned}
$$

Each $P_{n}$ is an $F_{\sigma}$-set and, by construction, the $P_{i}$ are mutually disjoint. We have $P_{i} \cap G_{n} \neq \varnothing$ for $n \geqslant i$. But note that for every $i$ the set $\left\{G_{n}: n \geqslant i\right\}$ must be a base for $U$, too, for otherwise $U$ would have an isolated point, which is impossible.

Now let $\left(q_{n}: n \geqslant 0\right)$ be an enumeration of the rationals. Define $f: E \rightarrow \mathbf{R}$ by $f\left(P_{n}\right)=\left\{q_{n}\right\}, n \geqslant 1$, and $f(E \backslash U)=\left\{q_{0}\right\}$. Obviously, $f$ is Borel measurable of class one. We prove that it is nearly continuous. Fix $x \in E$ and a neighborhood $W$ of $f(x)=q_{n}$. There exist numbers $n(1)<n(2)<\cdots$ such that $W \cap \mathbf{Q}=\left\{q_{n(i)}\right.$ : 
$i \in \mathbf{N}\}$. Consequently, we have $f^{-1}(W)=\bigcup\left\{P_{n(i)}: i \in \mathbf{N}\right\}$ if $q_{0} \notin W$ and $f^{-1}(W)=$ $(E \backslash U) \cup \cup\left\{P_{n(i)}: i>1\right\}$ if $q_{0} \in W$. In the first case this yields $\overline{f^{-1}(W)}=\bar{U}$ and in the second case $\overline{f^{-1}(W)}=E$. Therefore, $f$ is nearly continuous. It is clear that $\left(q_{n}\right)$ can be chosen such that $f$ is not continuous. Hence, we have arrived at a contradiction. This proves (I).

(II) Now we treat the general case. Assume that $E$ is not Baire and choose a nonempty open set $U$ which is of the first category. Let $C_{1}, C_{2}, \ldots$ be a sequence of closed nowhere dense sets with $C_{1} \subset C_{2} \subset C_{3} \subset \cdots$ and $\bigcup\left\{C_{n}: n \in \mathbf{N}\right\}=U$. As in part (I) we construct a sequence $\left(P_{n}\right)$ of mutually disjoint $F_{\sigma}$-subsets of $U$ with union $U$ and each $P_{n}$ dense in $U$. The mapping $f$ defined in (I) will then appear again and finish the proof. Let $\mathscr{B}_{n}, n \in \mathbf{N}$, be discrete sets of nonempty open sets such that $\mathscr{B}=\bigcup\left\{\mathscr{B}_{n}: n \in \mathbf{N}\right\}$ is a base for $U$. We construct sequences $(F(n): n \in \mathbf{N}),(H(n)$ : $n \in \mathbf{N}$ ) of subsets of $U$ with the following properties:

(a) $F(n), H(n)$ are $G_{\delta}$-sets as well as $F_{\sigma}$-sets;

(b) $F(n), H(n)$ are nowhere dense, $C_{n} \subset \bigcup\{F(i): i \leqslant n\} \cup \bigcup\{H(i): i \leqslant n\}$;

(c) for $n, m \in \mathbf{N}, n \neq m, F(n) \cap F(m)=\varnothing$ and $H(n) \cap H(m)=\varnothing$;

(d) $\bigcup\{F(n): n \in \mathbf{N}\} \cap \bigcup\{H(n): n \in \mathbf{N}\}=\varnothing$;

(e) each $F(n)$ is the union of a sequence $(F(n, i): i \in \mathbf{N})$ of $F_{\sigma}$-sets $F(n, i) \neq \varnothing$ such that $F(n, i) \cap F(n, j)=\varnothing$ for $i \neq j$ and such that $F(n, i) \cap B \neq \varnothing$ for all $B \in \mathscr{B}_{n}$.

Let $n=1$. For every $B \in \mathscr{B}_{1}$ there exists $n(B) \in \mathbf{N}$ such that $B \cap C_{n(B)}$ is an infinite set. For otherwise $B=\bigcup\left\{B \cap C_{n}: n \in \mathbf{N}\right\}$ would have to be countable, and therefore by (I) would be of the second category, contradicting our assumption. Now for every $B \in \mathscr{B}_{1}$ there exists a sequence $F(B, i), i \in \mathbf{N}$, of mutually disjoint nonempty $F_{\sigma}$-subsets of $B \cap C_{n(B)}$ with $\bigcup\{F(B, k): k \in \mathbf{N}\}=B \cap C_{n(B)}$. Now define $F(1, i):=\bigcup\left\{F(B, i): B \in \mathscr{B}_{1}\right\}$ and $F(1):=\bigcup\{F(1, i): i \in \mathbf{N}\}$. We have $F(1)=\bigcup\left\{B \cap C_{n(B)}: B \in \mathscr{B}_{1}\right\}$. Since $\mathscr{B}_{1}$ is discrete we derive that $F(1)$ is both a $G_{\delta}$ and an $F_{\sigma}$. Using the discreteness of $\mathscr{B}_{1}$ we also conclude that $F(1)$ is nowhere dense. Now define $H(1):=C_{1} \backslash F(1)$. Since $F(1)$ is a $G_{\delta}$ as well as an $F_{\sigma}$ the same is true for $H(1)$. Obviously, $H(1)$ is nowhere dense.

Suppose now that $F(i), H(i)$ have been defined for $i<n$ such that (a)-(e) are satisfied. For every $B \in \mathscr{B}_{n}$ there exists $n(B) \in \mathbf{N}$ such that

$$
(B \backslash(\bigcup\{F(i): i<n\} \cup \bigcup\{H(i): i<n\})) \cap C_{n(B)}=: X_{B}
$$

is infinite. For otherwise,

$$
B \backslash(\overline{\bigcup F(i): i<n\} \cup \bigcup\{H(i): i<n\}})
$$

would be a nonempty countable open subset of $U$, which again by (I) yields a contradiction. Choose nonempty and mutually disjoint $F_{\sigma}$-subsets $F(B, i)$ of $X_{B}$ with union $X_{B}$ and define $F(n, i):=\bigcup\left\{F(B, i): B \in \mathscr{B}_{n}\right\}$ and $F(n):=\bigcup\{F(n, i): i \in \mathbf{N}\}$. We have $F(n)=\bigcup\left\{X_{B}: B \in \mathscr{B}_{n}\right\}$ and this proves that $F(n)$ is nowhere dense and an $F_{\sigma}$ as well as a $G_{\delta}$. Now we define

$$
H(n):=C_{n} \backslash(\bigcup\{F(i): i \leqslant n\} \cup \bigcup\{H(i): i<n\}) .
$$

Then (a)-(e) are satisfied. 
Suppose now that $F(n), H(n), n \in \mathbf{N}$ have been constructed and define $P_{n}:=H(n) \cup \cup\{F(k, n): k \in \mathbf{N}\}, n \in \mathbf{N}$.

Next we prove that Theorem 5 does no longer hold true if the metrizability of $E$ is dropped.

Let $(E, \tau)$ be a topological space. There exists a topology $\tau_{0}$ on $E$, finer than $\tau$ such that the following hold true:

(1) $1_{E}:(E, \tau) \rightarrow\left(E, \tau_{0}\right)$ is nearly continuous,

(2) if $f:\left(E, \tau_{0}\right) \rightarrow F$ is nearly continuous, then it is in fact continuous (no matter what graph it has).

Proof. The proof uses the Kuratowski-Zorn lemma. Let $\mathfrak{M}$ be the set of all topologies $\sigma \supset \tau$ such that $1_{E}:(E, \tau) \rightarrow(E, \sigma)$ is nearly continuous. Define an order $\preceq$ on $\mathfrak{M}$ by the postulate that $\sigma_{1} \preceq \sigma_{2}$ if and only if $1_{E}:\left(E, \sigma_{1}\right) \rightarrow\left(E, \sigma_{2}\right)$ is nearly continuous and $\sigma_{1} \subset \sigma_{2}$. It is easily seen that every chain in $\mathfrak{M}$ has a supremum. Consequently, a maximal element $\tau_{0}$ of $\mathfrak{M}$ exists. Now we claim that every dense set in $\left(E, \tau_{0}\right)$ is open. Indeed, if $D$ is dense in $\left(E, \tau_{0}\right)$ then $\tau_{0} \cup\left\{D \cap W: W \in \tau_{0}\right\}$ is the base of a new topology $\tau_{1}$ on $E$ with $\tau_{0} \prec \tau_{1}$. Since $\tau_{0}$ is maximal we conclude $\tau_{0}=\tau_{1}$. Hence $D \in \tau_{0}$. But now we can derive (2) from this property of $\left(E, \tau_{0}\right)$. In fact, if $f:\left(E, \tau_{0}\right) \rightarrow F$ is nearly continuous then $D:=\left(E \backslash \overline{f^{-1}(U)}\right) \cup f^{-1}(U)$ is dense, hence open for open $U \subset F$. Thus $f^{-1}(U)=\operatorname{int} f^{-1}(U) \cap D$ is open.

Note that if we start with a first category space $(E, \tau)$ we will arrive at a first category space $\left(E, \tau_{0}\right)$. Hence we obtain 'many' counterexamples.

4. Almost continuous mappings. In [Wi] Wilhelm proves two graph theorems for almost continuous mappings, a concept which goes back to [BG] and seems to be closely related to the concept of nearly continuous mappings used here. $f: E \rightarrow F$ is called almost continuous if for every $x \in E$ and every neighborhood $U$ of $f(x)$ the set $D\left(f^{-1}(U)\right)$ is a neighborhood of $x$. Here $D(X)$ denotes the set of all $x \in E$ such that $X$ is of the second cateogry relative to $x$ (see [KM, p. 84]). The set $D(X)$ is always closed and contained in $\bar{X}$. Hence every almost continuous mapping is nearly continuous. (For details about the operator $D$ we refer to [KM, p. 428ff].) Wilhelm proves that:

Every almost continuous mapping $f$ from a Baire space $E$ to a regular space $F$ which is lower Baire (i.e. preimages of open sets are sets with the Baire property; see [Wi]) must be continuous. (See [Wi].)

In view of our Theorem 3 this makes us conjecture that 'almost continuity', although defined along quite similar lines, must be a far stronger property than 'nearly continuity'. This is corroborated by the following observation. Let $\mathfrak{M}$ be the model of set theory, constructed by Solovay $[\mathbf{J}]$, in which (i) the axiom of dependent choices holds, (ii) every set of reals is Lebesgue measurable, (iii) every set of reals has the Baire property. Consequently, in $\mathfrak{M}$ every mapping $f: \mathbf{R} \rightarrow \mathbf{R}$ is lower Baire. In view of Wilhelm's theorem this yields that:

In $\mathfrak{M}$ every almost continuous mapping $f: \mathbf{R} \rightarrow \mathbf{R}$ is continuous. 
This means that in the model $\mathfrak{M}$ we are not able to find an almost continuous real mapping which is not continuous. However, it is easy to find nearly continuous mappings $f: \mathbf{R} \rightarrow \mathbf{R}$ which are not continuous. Take for example $f(x)=0$ for $x$ rational and $f(x)=1$ for $x$ irrational, then $f$ is nearly continuous but not continuous. Note that in view of Theorem $4 f$ cannot be of the first Baire class.

\section{BIBLIOGRAPHY}

[BG] J. C. Bradford and C. Goffman, Metric spaces in which Blumberg's Theorem holds, Proc. Amer. Math. Soc. 11 (1960), 667-670.

[BP] T. Byczkowski and R. Pol, On the closed graph and open mapping theorems, Bull. Acad. Polon. Sci. Sér. Sci. Math. 24 (1976), 723-726.

[CČN] J. Chaber, M. M. Čoban and K. Nagami, On monotonic generalizations of Moore spaces, Čech-complete spaces and p-spaces, Fund. Math. 84 (1974), 107-119.

[Ce] J. G. Ceder, Some generalizations of metric spaces, Pacific J. Math. 11 (1961), 105-125.

[E] R. Engelking, General topology, PWN, Warszawa, 1976.

[Hu] T. Husain, Introduction to topological groups, Saunders, Philadelphia, Pa., 1966.

[J] T. Jech, Introduction to set theory, Lecture Notes in Math., no. 217, Springer-Verlag, Berlin and New York, 1971.

[Ke] J. L. Kelley, General topology, Van Nostrand, New York, 1955.

[Kö] G. Köthe, Topological vector spaces. II, Springer-Verlag, New York, 1979.

[Ku] K. Kuratowski, Topology. I, Academic Press, New York, 1966.

[KM] K. Kuratowski and A. Mostowski, Set theory, PWN, Warszawa, 1968.

[LR] V. L. Levin and D. A. Raịkow, Closed graph theorems for uniform spaces, Soviet Math. Dokl. 4 (1963), $791 \mathrm{ff}$.

[Ox] J. C. Oxtoby, The Banach-Mazur game and Banach category theorem, Contribution to the Theory of Games, vol. 3, Ann. of Math. Studies, no. 39, Princeton Univ. Press, Princeton, N. J., 1957.

[We] J. D. Weston, On the comparison of topologies, J. London Math. Soc. 32 (1957), $342 \mathrm{ff}$.

[Wh] H. E. White, Topological spaces that are $\alpha$-favourable for a player with perfect information, Proc. Amer. Math. Soc. 50 (1975), 477-482.

[Wi] M. Wilhelm, Almost lower semicontinuous multifunctions and the Souslin-graph theorem, Comment. Math. Univ. Carolinae 23 (1982), 147-158.

[WW] H. H. Wicke and J. M. Worrell, On the open continuous images of paracompact Čech complete spaces, Pacific. J. Math. 37 (1971), 265-275.

[WW1] _ Characterizations of developable topological spaces, Canad. J. Math. 17 (1965), 820-830.

Mathematisches Institut B, Universität Stuttgart, Pfaffenwaldring, Federal Republic of GERMANY

Current address: Gollenstrasse 13, 7300 Esslingen, Federal Republic of Germany 